Portland State University

PDXScholar

$10-1-2005$

\title{
Dependence, Dispersiveness, and Multivariate Hazard Rate Ordering
}

\author{
Baha-Eldin Khaledi \\ Razi University \\ Subhash C. Kochar \\ Portland State University, kochar@pdx.edu
}

Follow this and additional works at: https://pdxscholar.library.pdx.edu/mth_fac

Part of the Other Mathematics Commons

Let us know how access to this document benefits you.

\section{Citation Details}

Khaledi, E. and Kochar, S. (2005). Dependence, dispersiveness, and multivariate hazard rate ordering. Probability in the Engineering and Informational Sciences, 19, pp 427-446.

This Article is brought to you for free and open access. It has been accepted for inclusion in Mathematics and Statistics Faculty Publications and Presentations by an authorized administrator of PDXScholar. Please contact us if we can make this document more accessible: pdxscholar@pdx.edu. 


\title{
DEPENDENCE, DISPERSIVENESS, AND MULTIVARIATE HAZARD RATE ORDERING
}

\author{
BAHA-ELDIN KHALEDI \\ Statistical Research Center \\ Tehran, Iran \\ and \\ Department of Statistics \\ College of Sciences \\ Razi University \\ Kermanshah, Iran \\ E-mail: bkhaledi@hotmail.com \\ SUBHASH KochaR \\ Department of Mathematics and Statistics \\ Portland State University \\ Portland, Oregon 97201 \\ E-mail: subhash.kochar@gmail.com
}

\begin{abstract}
To compare two multivariate random vectors of the same dimension, we define a new stochastic order called upper orthant dispersive ordering and study its properties. We study its relationship with positive dependence and multivariate hazard rate ordering as defined by Hu, Khaledi, and Shaked (Journal of Multivariate Analysis, 2002). It is shown that if two random vectors have a common copula and if their marginal distributions are ordered according to dispersive ordering in the same direction, then the two random vectors are ordered according to this new upper orthant dispersive ordering. Also, it is shown that the marginal distributions of two upper orthant dispersive ordered random vectors are also dispersive ordered. Examples and applications are given.
\end{abstract}

\section{INTRODUCTION}

It is of interest to compare two random variables in terms of their variability. Although this topic has been studied extensively in the univariate case, several attempts have been made to extend it to the multivariate case. Important contributions in this case 
have been made by Giovagnoli and Wynn [8], Shaked and Shanthikumar [21], and Fernandez-Ponce and Suarez-Llorens [7], among others.

Let $X$ and $Y$ be two univariate random variables with distribution functions $F$ and $G$ and with survival functions $\bar{F}$ and $\bar{G}$, respectively. A basic concept for comparing variability in distributions is that of dispersive ordering. $X$ is said to be less dispersed than $Y$ (denoted by $\left.X \leq_{\text {disp }} Y\right)$ if

$$
F^{-1}(\beta)-F^{-1}(\alpha) \leq G^{-1}(\beta)-G^{-1}(\alpha) \quad \text { whenever } 0<\alpha \leq \beta<1,
$$

where $F^{-1}$ and $G^{-1}$ are the right continuous inverses of the distribution functions $F$ and $G$, respectively. This means that the difference between any two quantiles of $X$ is smaller than the difference between the corresponding quantiles of $Y$. In case the random variables $X$ and $Y$ are of continuous type with hazard rates $r_{F}$ and $r_{G}$, respectively, then $X \leq_{\text {disp }} Y$ if and only if

$$
r_{G}\left(G^{-1}(p)\right) \leq r_{F}\left(F^{-1}(p)\right), \quad \forall p \in[0,1]
$$

For more details on dispersive ordering, see Shaked and Shanthikumar [20, Sec. 2B].

In analogy with the characterization (1.2) of the univariate dispersive ordering, we introduce a new order in the multivariate case, which we call upper orthant dispersive ordering and study its properties. According to (1.2), $X \leq_{\text {disp }} Y$ if and only if the hazard rates of $X$ and $Y$ at the quantiles of the same order $p$ are ordered for all values of $p \in[0,1]$. To this end, we first recall the definition of hazard rate (or hazard gradient) in the multivariate case. Consider a random vector $\mathbf{X}=$ $\left(X_{1}, \ldots, X_{n}\right)$ with a partially differentiable survival function $\bar{F}(\mathbf{x})=P\{\mathbf{X}>\mathbf{x}\}$. The function $R=-\log \bar{F}$ is called the hazard function of $\mathbf{X}$, and the vector $\mathbf{r}_{\mathbf{X}}$ of partial derivatives, defined by

$$
\mathbf{r}_{\mathbf{X}}(\mathbf{x})=\left(r_{\mathbf{X}}^{(1)}(\mathbf{x}), \ldots, r_{\mathbf{X}}^{(n)}(\mathbf{x})\right)=\left(\frac{\partial}{\partial x_{1}} R(\mathbf{x}), \ldots, \frac{\partial}{\partial x_{n}} R(\mathbf{x})\right)
$$

for all $\mathbf{x} \in\{\mathbf{x}: \bar{F}(\mathbf{x})>0\}$, is called the hazard gradient of $\mathbf{X}$ (see Johnson and Kotz [11] and Marshall [15]). Note that $r_{\mathbf{X}}^{(i)}(\mathbf{x})$ can be interpreted as the conditional hazard rate of $X_{i}$ evaluated at $x_{i}$, given that $X_{j}>x_{j}$ for all $j \neq i$; that is,

$$
r_{\mathbf{X}}^{(i)}(\mathbf{x})=\frac{f_{i}\left(x_{i} \mid \bigcap_{j \neq i}\left\{X_{j}>x_{j}\right\}\right)}{\bar{F}_{i}\left(x_{i} \mid \bigcap_{j \neq i}\left\{X_{j}>x_{j}\right\}\right)},
$$

where $f_{i}\left(\cdot \mid \bigcap_{j \neq i}\left\{X_{j}>x_{j}\right\}\right)$ and $\bar{F}_{i}\left(\cdot \mid \bigcap_{j \neq i}\left\{X_{j}>x_{j}\right\}\right)$ are respectively the conditional density and the conditional survival functions of $X_{i}$, given that $X_{j}>x_{j}$ for all $j \neq i$. For convenience, here and below we set $r_{\mathbf{X}}^{(i)}(\mathbf{x})=\infty$ for all $\mathbf{x} \in\{\mathbf{x}: \bar{F}(\mathbf{x})=0\}$. Now, we define upper orthant dispersive ordering. 
Definition 1.1: Let $\mathbf{X}=\left(X_{1}, \ldots, X_{n}\right)$ and $\mathbf{Y}=\left(Y_{1}, \ldots, Y_{n}\right)$ be two random vectors with respective survival functions $\bar{F}$ and $\bar{G}$. We say that $\mathbf{X}$ is smaller than $\mathbf{Y}$ according to upper orthant dispersive ordering (denoted by $\mathbf{X} \stackrel{\text { uo-disp }}{\lessgtr} \mathbf{Y}$ ) if for all $u_{j} \in$ $[0,1], j=1, \ldots, n, j \neq i$,

$$
\left(X_{i} \mid \bigcap_{j \neq i}\left\{X_{j}>F_{j}^{-1}\left(u_{j}\right)\right\}\right) \leq_{\operatorname{disp}}\left(Y_{i} \mid \bigcap_{j \neq i}\left\{Y_{j}>G_{j}^{-1}\left(u_{j}\right)\right\}\right),
$$

for $i=1, \ldots, n$.

In case the distributions under consideration are absolutely continuous, the upper orthant dispersive ordering can be equivalently expressed in terms of the hazard gradients at the quantiles of the same orders of the conditional distributions. If we denote by $x_{i}(\beta ; \mathbf{u})$ and $y_{i}(\beta ; \mathbf{u})$, the $\beta$ th quantiles of the conditional distributions $\left(X_{i} \mid \bigcap_{j \neq i}\left\{X_{j}>F_{j}^{-1}\left(u_{j}\right)\right\}\right)$ and $\left(Y_{i} \mid \bigcap_{j \neq i}\left\{Y_{j}>G_{j}^{-1}\left(u_{j}\right)\right\}\right)$, respectively, then

$$
\begin{aligned}
\mathbf{X} \stackrel{\text { uo-disp }}{\lessgtr} \mathbf{Y} & \Leftrightarrow r_{\mathbf{X}}^{(i)}\left(F_{1}^{-1}\left(u_{1}\right), \ldots, F_{i-1}^{-1}\left(u_{i-1}\right), x_{i}(\beta ; \mathbf{u}), \ldots, F_{n}^{-1}\left(u_{n}\right)\right) \\
& \geq r_{\mathbf{Y}}^{(i)}\left(G_{1}^{-1}\left(u_{1}\right), \ldots, G_{i-1}^{-1}\left(u_{i-1}\right), y_{i}(\beta ; \mathbf{u}), \ldots, G_{n}^{-1}\left(u_{n}\right)\right),
\end{aligned}
$$

for every $\beta \in[0,1], \mathbf{u} \in[0,1]^{n-1}$, and $i=1, \ldots, n$, where $r_{\mathbf{X}}^{(i)}$ and $r_{\mathbf{Y}}^{(i)}$ stand for the $i$ th components of the hazard gradients of $\mathbf{X}$ and $\mathbf{Y}$, respectively.

The following slightly modified version of a theorem of Saunders and Moran [19] provides a useful tool for establishing dispersive ordering among members of a parametric family of distributions.

THEOREM 1.1: Let $X_{a}$ be a random variable with distribution function $F_{a}$ for each $a \in R$ such that the following hold:

(i) $F_{a}$ is supported on some interval $\left(x_{-}^{(a)}, x_{+}^{(a)}\right) \subseteq(-\infty, \infty)$ and has density $f_{a}$ that does not vanish on any subinterval of $\left(x_{-}^{(a)}, x_{+}^{(a)}\right)$.

(ii) The derivative of $F_{a}$ with respect to a exists and is denoted by $F_{a}^{\prime}$.

Then

$$
X_{a} \geq_{\mathrm{disp}} X_{a^{*}} \text { for } a, a^{*} \in R, \text { and } a>a^{*},
$$

if and only if,

$$
F_{a}^{\prime}(x) / f_{a}(x) \text { is decreasing in } x
$$

In the next example we identify conditions under which two bivariate normal random vectors are ordered according to upper orthant dispersive ordering. More examples are discussed in Section 4. 
Example 1.1 (Bivariate Normal Distribution): Let $\mathbf{X}$ and $\mathbf{Y}$ follow bivariate Normal distributions, each with mean vector $(0,0)$ and with dispersion matrices

$$
\boldsymbol{\Sigma}^{X}=\left(\begin{array}{cc}
\sigma_{1}^{2} & \rho \sigma_{1} \sigma_{2} \\
\rho \sigma_{1} \sigma_{2} & \sigma_{2}^{2}
\end{array}\right) \quad \text { and } \quad \boldsymbol{\Sigma}^{Y}=\left(\begin{array}{cc}
\sigma_{1}^{2} & \rho^{\prime} \sigma_{1} \sigma_{2} \\
\rho^{\prime} \sigma_{1} \sigma_{2} & \sigma_{2}^{2}
\end{array}\right),
$$

respectively, with $\sigma_{i}>0$ for $i=1,2$. For the time being, we are assuming that the marginal distributions of $\mathbf{X}$ and $\mathbf{Y}$ are identical. The general case is considered later. We use Theorem 1.1 to prove that in case $\rho$ and $\rho^{\prime}$ are of the same sign, then

$$
\left|\rho^{\prime}\right| \leq|\rho| \Rightarrow \mathbf{X} \stackrel{\text { uo-disp }}{\lessgtr} \mathbf{Y} .
$$

Let us denote by $G_{\rho}$, the distribution function of $\left\{X_{1} \mid X_{2}>y\right\}$ (we are suppressing its dependence on $y$ for the clarity of notation). Then

$$
\begin{aligned}
G_{\rho}(x) & =\frac{1}{P\left(X_{2}>y\right)} \int_{-\infty}^{x} \int_{y}^{+\infty} f_{X_{1}, X_{2}}(u, v) d u d v \\
& =\frac{1}{P\left(X_{2}>y\right)} \int_{y}^{+\infty} P\left(X_{1} \leq x \mid X_{2}=v\right) f_{X_{2}}(v) d v \\
& =\frac{1}{P\left(X_{2}>y\right)} \int_{y}^{+\infty} \Phi\left(\frac{x-\frac{\rho \sigma_{1}}{\sigma_{2}} v}{\sigma_{1}\left(1-\rho^{2}\right)^{1 / 2}}\right) \frac{1}{\sigma_{2}} \phi\left(\frac{v}{\sigma_{2}}\right) d v
\end{aligned}
$$

and the corresponding conditional density function is

$$
g_{\rho}(x)=\frac{1}{P\left(X_{2}>y\right) \sigma_{1}\left(1-\rho^{2}\right)^{1 / 2}} \int_{y}^{+\infty} \phi\left(\frac{x-\frac{\rho \sigma_{1}}{\sigma_{2}} v}{\sigma_{1}\left(1-\rho^{2}\right)^{1 / 2}}\right) \frac{1}{\sigma_{2}} \phi\left(\frac{v}{\sigma_{2}}\right) d v .
$$

Now we compute

$$
\begin{aligned}
G_{\rho}^{\prime}(x)= & \frac{\partial}{\partial \rho} G_{\rho}(x) \\
= & \frac{1}{P\left(X_{2}>y\right)} \int_{y}^{+\infty}\left\{\frac{-\frac{\sigma_{1}}{\sigma_{2}} v\left(\sigma_{1}\left(1-\rho^{2}\right)^{1 / 2}\right)+\frac{\sigma_{1} \rho}{\left(1-\rho^{2}\right)^{1 / 2}}\left(x-\frac{\rho \sigma_{1}}{\sigma_{2}} v\right)}{\sigma_{1}^{2}\left(1-\rho^{2}\right)}\right\} \\
& \times \phi\left(\frac{x-\frac{\rho \sigma_{1}}{\sigma_{2}} v}{\sigma_{1}\left(1-\rho^{2}\right)^{1 / 2}}\right) \frac{1}{\sigma_{2}} \phi\left(\frac{v}{\sigma_{2}}\right) d v,
\end{aligned}
$$


which simplifies to

$$
\begin{aligned}
G_{\rho}^{\prime}(x)= & \frac{e^{-\left(x^{2} / 2 \sigma_{1}^{2}\right)}}{P\left(X_{2}>y\right) \sqrt{2 \pi}} \int_{y}^{+\infty} \frac{\left(\frac{\rho \sigma_{2}}{\sigma_{1}}-v\right)}{\sqrt{2 \pi} \sigma_{2}^{2}\left(1-\rho^{2}\right)^{3 / 2}} \\
& \times \exp \left(-\frac{1}{2 \sigma_{2}^{2}\left(1-\rho^{2}\right)}\left(v-\frac{\rho x \sigma_{2}}{\sigma_{1}}\right)^{2}\right) d v \\
= & -\frac{e^{-\left(x^{2} / 2 \sigma_{1}^{2}\right)}}{P\left(X_{2}>y\right)(2 \pi)\left(1-\rho^{2}\right)^{1 / 2}} \exp \left(-\frac{1}{2 \sigma_{2}^{2}\left(1-\rho^{2}\right)}\left(y-\frac{\rho x \sigma_{2}}{\sigma_{1}}\right)^{2}\right) .
\end{aligned}
$$

Similarly, the conditional density function $g_{\rho}(x)$ can be written as

$$
\begin{aligned}
g_{\rho}(x)= & \frac{e^{-\left(x^{2} / 2 \sigma_{1}^{2}\right)}}{P\left(X_{2}>y\right) \sqrt{2 \pi} \sigma_{1}} \int_{y}^{+\infty} \frac{1}{\sigma_{2}\left(1-\rho^{2}\right)^{1 / 2}(2 \pi)^{1 / 2}} \\
& \times \exp \left(-\frac{1}{2 \sigma_{2}^{2}\left(1-\rho^{2}\right)}\right)\left(v-\frac{\rho x \sigma_{2}}{\sigma_{1}}\right)^{2} d v .
\end{aligned}
$$

This gives

$$
h(x)=\frac{G_{\rho}^{\prime}(x)}{g_{\rho(x)}}=-\sigma_{2} \sigma_{1} r_{W_{x}}(y),
$$

where $r_{W_{x}}$ (.) denotes the hazard rate of $W_{x}$, a normal random variable with mean $\rho x \sigma_{2} / \sigma_{1}$ and variance $\sigma_{2}^{2}\left(1-\rho^{2}\right)$. It is known that the family of normal random variables with a fixed variance but with different means is ordered according to hazard rate order and the one with the smaller mean has the greater hazard rate. Using this fact, it follows that if $\rho>0$, then $h(x)$ is increasing in $x$. Hence, by Theorem 1.1, $0 \leq \rho^{\prime} \leq \rho \Rightarrow \mathbf{X} \stackrel{\text { uo-disp }}{\lessgtr}$ Y. If $\rho<0$, then $h(x)$ is decreasing in $x$; hence, $\left(X_{1}, X_{2}\right)$ is increasing in $\rho$ in the sense of upper orthant dispersive ordering. This proves the required result.

The organization of the article is as follows. In Section 2 we study some properties of the upper orthant dispersive ordering as defined earlier. It is proved that if two random vectors have the same dependence structure (copula), then they are ordered according to upper orthant dispersive ordering if and only if their corresponding marginals are ordered according to univariate dispersive ordering. In Section 3 we consider the special case of nonnegative random variables (more generally, if the conditional distributions have common left end points of their supports). It is shown that if two random vectors have the same marginal distributions and they are ordered according to upper orthant dispersive ordering, then their bivariate copulas 
are ordered, implying that one random vector is more dependent in the sense of positive quadrant dependence than the other. We also study the connection between upper orthant dispersive ordering and multivariate hazard rate ordering as introduced by $\mathrm{Hu}$, Khaledi, and Shaked [9]. The last section is devoted to some examples and applications. It is shown that if two univariate distributions are ordered according to dispersive ordering, then the corresponding vectors of order statistics from them are ordered according to upper orthant dispersive ordering.

\section{PROPERTIES OF UPPER ORTHANT DISPERSIVE ORDERING}

In this section we establish an interesting property of the upper orthant dispersive ordering that if two $n$-dimensional random vectors $\mathbf{X}$ and $\mathbf{Y}$ have the same dependence structure in the sense that they have the same copula, then dispersive ordering among the marginal distributions implies upper orthant dispersive ordering and vice versa. The notion of copula has been introduced by Sklar [23], and studied by, among others, Kimeldrof and Sampson [13] under the name of uniform representation and by Deheuvels [5] under the name of dependence function. A copula $C$ is a cumulative distribution function with uniform margins on $[0,1]$. Given a copula $C$, if one defines

$$
F(\mathbf{x})=C\left(F_{1}\left(x_{1}\right), F_{2}\left(x_{2}\right), \ldots F_{n}\left(x_{n}\right)\right), \quad \mathbf{x} \in \mathbb{R}^{n},
$$

then $F$ is a multivariate distribution function with margins as $F_{1}, F_{2}, \ldots, F_{n}$. For any multivariate distribution function $F$ with margins as $F_{1}, F_{2}, \ldots, F_{n}$, there exists a copula $C$ such that (2.1) holds. If $F$ is continuous, then $C$ is unique and can be constructed as follows:

$$
C(\mathbf{u})=F\left[F_{1}^{-1}\left(u_{1}\right), F_{2}^{-1}\left(u_{2}\right), \ldots, F_{n}^{-1}\left(u_{n}\right)\right], \quad \mathbf{u} \in[0,1]^{n} .
$$

It follows that if $\mathbf{X}$ and $\mathbf{Y}$ are two $n$-dimensional random vectors with margins as $\left(F_{1}, F_{2}, \ldots, F_{n}\right)$ and $\left(G_{1}, G_{2}, \ldots, G_{n}\right)$, respectively, and if they have the same copula, then

$$
\left(F_{1}\left(X_{1}\right), F_{2}\left(X_{2}\right), \ldots F_{n}\left(X_{n}\right)\right) \stackrel{\text { st }}{=}\left(G_{1}\left(Y_{1}\right), G_{2}\left(Y_{2}\right), \ldots G_{n}\left(Y_{n}\right)\right)
$$

For $i=1, \ldots, n$, let us denote by $H_{i, \mathbf{u}}^{X}$ the cumulative distribution function (cdf) of the conditional distribution $\left(X_{i} \mid \bigcap_{j \neq i}\left\{X_{j}>F_{j}^{-1}\left(u_{j}\right)\right\}\right)$ and by $H_{i, \mathbf{u}}^{Y}$ that of $\left(Y_{i} \mid \cap_{j \neq i}\left\{Y_{j}>G_{j}^{-1}\left(u_{j}\right)\right\}\right)$. To prove the next theorem, we first prove the following lemma, which may be of independent interest.

Lemma 2.1: If two n-dimensional random vectors $\mathbf{X}$ and $\mathbf{Y}$ have the same copula, then, for $i=1, \ldots n$,

$$
F_{i} \circ H_{i, \mathbf{u}}^{X}(\beta)=G_{i} \circ H_{i, \mathbf{u}}^{X^{-1}}(\beta), \quad \beta \in[0,1], \mathbf{u} \in[0,1]^{n-1} .
$$


Proof: Proving (2.4) is equivalent to proving that for $i=1, \ldots n$ and $\mathbf{u} \in[0,1]^{n-1}$,

$$
\begin{aligned}
\bar{H}_{i, \mathbf{u}}^{X} \circ F_{i}^{-1}(v) & =\bar{H}_{i, \mathbf{u}}^{Y} \circ G_{i}^{-1}(v), \quad \forall v \in[0,1] \\
& \Leftrightarrow P\left[X_{i}>F_{i}^{-1}(v) \mid \bigcap_{j \neq i}\left\{X_{j}>F_{j}^{-1}\left(u_{j}\right)\right\}\right] \\
& \left.=P\left[Y_{i}>G_{i}^{-1}(v) \mid \bigcap_{j \neq i}\left\{Y_{j}>G_{j}^{-1}\left(u_{j}\right)\right\}\right]\right], \quad \forall v \in[0,1] \\
& \Leftrightarrow P\left[F_{i}\left(X_{i}\right)>v \mid \bigcap_{j \neq i}\left\{F_{j}\left(X_{j}\right)>u_{j}\right\}\right] \\
& =P\left[G_{i}\left(Y_{i}\right)>v \mid \bigcap_{j \neq i}\left\{G_{j}\left(Y_{j}\right)>u_{j}\right\}\right], \quad \forall v \in[0,1],
\end{aligned}
$$

which is true because of (2.3), since $\mathbf{X}$ and $\mathbf{Y}$ have the same copula.

TheOREM 2.1: Let $\mathbf{X}$ and $\mathbf{Y}$ be two $n$-dimensional random vectors with the same copula. Then $\mathbf{X} \stackrel{\text { uo-disp }}{\lessgtr} \mathbf{Y}$ if and only if $X_{i} \leq_{\text {disp }} Y_{i}, i=1, \ldots, n$.

Proof: By definition,

$$
\begin{gathered}
\mathbf{X} \stackrel{\text { uo-disp }}{\lessgtr} \mathbf{Y} \Leftrightarrow H_{i, \mathbf{u}}^{Y-1}(\beta)-H_{i, \mathbf{u}}^{X}(\beta) \text { is increasing in } \beta \in[0,1], \\
\mathbf{u} \in[0,1]^{n-1}, \text { for } i=1, \ldots, n .
\end{gathered}
$$

It follows from (2.4) that if $\mathbf{X}$ and $\mathbf{Y}$ have the same copula, then for $i=1, \ldots n$ and $\mathbf{u} \in[0,1]^{n-1}$,

$$
H_{i, \mathbf{u}}^{Y^{-1}}(\beta)-H_{i, \mathbf{u}}^{X^{-1}}(\beta)=G_{i}^{-1} \circ F_{i}\left(H_{i, \mathbf{u}}^{X^{-1}}(\beta)\right)-H_{i, \mathbf{u}}^{X^{-1}}(\beta),
$$

for $i=1, \ldots n$ and for every $\beta \in[0,1]$.

It is easy to see that the right-hand side of (2.6) is increasing in $\beta$ if and only if $G_{i}^{-1} F_{i}(x)-x$ is increasing in $x$ (i.e., if and only if $X_{i} \leq_{\text {disp }} Y_{i}, i=1, \ldots, n$ ). This proves the desired result.

Recently, Müller and Scarsini [16] have investigated some other multivariate stochastic orders for which results parallel to Theorem 2.1 hold for those orders. The following interesting property of the upper orthant dispersive ordering immediately follows from Theorem 2.1.

Corollary 2.1: Let $\mathbf{Y}=\left(a_{1} X_{1}+b_{1}, a_{2} X_{2}+b_{2}, \ldots, a_{n} X_{n}+b_{n}\right)$. Then for $a_{i} \geq 1$, $b_{i} \in \mathbb{R}, \mathbf{X} \stackrel{\text { uo-disp }}{\lessgtr} \mathbf{Y}$.

Proof: Since $\mathbf{X}$ and $\mathbf{Y}$ have the same copula, the required result follows immediately from Theorem 2.1 . 
Example 2.1 (Multivariate Normal Distributions): Let $\mathbf{X}$ follow the $p$-variate multivariate Normal distribution with mean vector $\boldsymbol{\mu}$ and dispersion matrix $\boldsymbol{\Sigma}=\left(\left(\sigma_{i j}\right)\right)$, with $\sigma_{i j}=\rho_{i j} \sigma_{i} \sigma_{j}, \rho_{i i}=1, \sigma_{i}>0$, and $i, j=1, \ldots, p$. Let $\mathbf{Y}$ follow the $p$-variate multivariate Normal distribution with mean vector $\boldsymbol{\mu}^{\prime}$ and dispersion matrix $\boldsymbol{\Sigma}^{\prime}=$ $\left(\left(\sigma_{i j}^{\prime}\right)\right)$, with $\sigma_{i j}^{\prime}=\rho_{i j} \sigma_{i}^{\prime} \sigma_{j}^{\prime}, \sigma_{i}^{\prime}>0$, and $i, j=1, \ldots, p$. It is known that $\mathbf{X}$ and $\mathbf{Y}$ have the same copula. It follows from Theorem 2.1 that $\mathbf{X} \stackrel{\text { uo-disp }}{\lessgtr} \mathbf{Y}$ if and only if $\sigma_{i} \leq \sigma_{i}^{\prime}$ for $i=1, \ldots, p$. This result in conjunction with Example 1.1 leads us to the following result for comparing two bivariate Normal distributions.

Let $\mathbf{X}$ and $\mathbf{Y}$ follow bivariate Normal distributions with dispersion matrices

$$
\Sigma=\left(\begin{array}{cc}
\sigma_{1}^{2} & \rho \sigma_{1} \sigma_{2} \\
\rho \sigma_{1} \sigma_{2} & \sigma_{2}^{2}
\end{array}\right), \quad \boldsymbol{\Sigma}^{\prime}=\left(\begin{array}{cc}
\sigma_{1}^{\prime 2} & \rho^{\prime} \sigma_{1}^{\prime} \sigma_{2}^{\prime} \\
\rho^{\prime} \sigma_{1}^{\prime} \sigma_{2}^{\prime} & \sigma_{2}^{\prime 2}
\end{array}\right),
$$

respectively. If $0<\sigma_{i} \leq \sigma_{i}^{\prime}$ for $i=1,2$ and $\left|\rho^{\prime}\right| \leq|\rho|<1$, then $\mathbf{X} \stackrel{\text { uo-disp }}{\leqslant} \mathbf{Y}$.

It will be interesting to find necessary and sufficient conditions under which two multivariate normal random vectors will be ordered according to upper orthant dispersive ordering in the general case.

It follows immediately that if two random vectors are ordered according to upper orthant dispersive ordering, then so are their corresponding subsets. In particular, their marginal distributions will be then ordered according to univariate dispersive ordering.

Theorem 2.2: Let $\mathbf{X}$ and $\mathbf{Y}$ be two n-dimensional random vectors such that $\mathbf{X} \stackrel{\text { uo-disp }}{\lessgtr} \mathbf{Y}$. Then

$$
\mathbf{X}_{I} \stackrel{\text { uo-disp }}{\lessgtr} \mathbf{Y}_{I}
$$

where $I=\left\{i_{1}, i_{2}, \ldots, i_{k}\right\} \subset\{1,2, \ldots, n\}, \mathbf{X}_{I}=\left(X_{i_{1}}, \ldots, X_{i_{k}}\right), \mathbf{Y}_{I}=\left(Y_{i_{1}}, \ldots, Y_{i_{k}}\right)$, and $k=1, \ldots, n$.

The proof of the next result is also immediate.

TheOREm 2.3: Let $\mathbf{X}_{1}, \ldots, \mathbf{X}_{m}$ be a set of independent random vectors for which the dimension of $\mathbf{X}_{i}$ is $k_{i}, i=1, \ldots, m$. Let $\mathbf{Y}_{1}, \ldots, \mathbf{Y}_{m}$ be another set of independent random variables for which the dimension of $\mathbf{Y}_{i}$ is $k_{i}, i=1, \ldots, m$. Then

$$
\left(\mathbf{X}_{i} \stackrel{\text { uo-disp }}{\lessgtr} \mathbf{Y}_{i}, i=1, \ldots, m\right) \Rightarrow\left(\mathbf{X}_{1}, \ldots, \mathbf{X}_{m}\right) \stackrel{\text { uo-disp }}{\lessgtr}\left(\mathbf{Y}_{1}, \ldots, \mathbf{Y}_{m}\right) .
$$

Remark 2.1: A consequence of (2.7) is that if $X_{1}, \ldots, X_{n}$ is a collection of independent univariate random variables and $Y_{1}, \ldots, Y_{n}$ is another set of independent random variables, then $X_{i} \leq_{\text {disp }} Y_{i}, i=1, \ldots, n$ implies $\mathbf{X} \stackrel{\text { uo-disp }}{\lessgtr} \mathbf{Y}$.

In general, there does not seem to be any direct connection between upper orthant dispersive ordering and the multivariate dispersive ordering as introduced by Fernandez-Ponce and Suarez-Llorens [7]. According to their definition, $\mathbf{X} \leq_{\text {disp }}$ Y may not imply that $X_{i} \leq_{\text {disp }} Y_{i}$ for $i=1, \ldots, n$. Also, the multivariate dispersive ordering as defined by them may not be preserved under permutations of the vari- 
ables. On the other hand, the upper orthant dispersive ordering is invariant under the same permutation of the two vectors and their marginals are also ordered according to univariate dispersive ordering. Obviously, if $\mathbf{X} \stackrel{\text { uo-disp }}{\lessgtr} \mathbf{Y}$, then $\operatorname{tr} \Sigma_{X} \leq \operatorname{tr} \Sigma_{Y}$, where $\Sigma_{X}$ and $\Sigma_{Y}$ denote the dispersion matrices of $\mathbf{X}$ and $\mathbf{Y}$, respectively.

\section{THE CASE OF NONNEGATIVE RANDOM VARIABLES}

In this section, we will restrict our attention to the case in which the random vectors under consideration are nonnegative or, more generally, they have a finite common left end point of their supports. We will see that certain results hold in this case that may not hold in the general case. The following assumption will be made at some places in this article.

Assumption A: The random variables $\left\{X_{i} \mid \bigcap_{j \neq i}\left\{X_{j}>F_{j}^{-1}\left(u_{j}\right)\right\}\right\}$ and $\left\{Y_{i} \mid \cap_{j \neq i}\left\{Y_{j}>G_{j}^{-1}\left(u_{j}\right)\right\}\right\}$ have a finite common left endpoint of their supports for all $\boldsymbol{u}$ and for $i=1, \ldots, n$.

In the univariate case, for nonnegative random variables, there is an intimate connection between hazard rate ordering and dispersive ordering and which is made more explicit in the following result of Bagai and Kochar [1]. We use this theorem to prove some of the results of this section.

THEOREM 3.1: Let $X$ and $Y$ be two univariate random variables with distribution functions $F$ and $G$, respectively, such that $F(0)=G(0)=0$. Then the following hold:

(a) If $Y \leq_{\mathrm{hr}} X$ and either F or $G$ is DFR (decreasing failure rate), then $Y \leq_{\mathrm{disp}} X$.

(b) If $Y \leq_{\mathrm{disp}} X$ and either F or $G$ is IFR (increasing failure rate), then $Y \leq_{\mathrm{hr}} X$.

For a bivariate random vector $(S, T)$, we say that $T$ is right tail increasing in $S$ if $P[T>t \mid S>s]$ is increasing in $s$ for all $t$, and we denote this relationship by $\operatorname{RTI}(T \mid S)$. If $S$ and $T$ are continuous lifetimes, then $T$ is right tail increasing in $S$ if and only if $r(s \mid T>t) \leq r(s \mid T>0)=r_{S}(s)$ for all $s>0$ and for each fixed $t$. The RTI property is weaker than the RCSI (right corner set increasing) property, but stronger than PQD (positive quadrant dependence). In the next theorem, we study the effect of positive dependence on upper orthant dispersive ordering for nonnegative random vectors.

THEOREM 3.2: Let $\mathbf{X}=\left(X_{1}, X_{2}\right)$ be a bivariate random vector such that the left end point of the support of $\left\{X_{i} \mid X_{j}>F_{j}^{-1}(u)\right\}$ is finite and independent of $u \in[0,1]$ for $i, j=1,2$. Let $\mathbf{X}^{\mathbf{I}}=\left(X_{1}^{I}, X_{2}^{I}\right)$ be a random vector of independent random variables such that $X_{i} \stackrel{\text { st }}{=} X_{i}^{I}, i=1,2$.

(a) If $X_{i}$ is RTI in $X_{j}, i \neq j$, and $X_{i}$ is DFR for $i, j=1,2$, then

$$
\left(X_{1}, X_{2}\right) \stackrel{\text { uo-disp }}{\lessgtr}\left(X_{1}^{I}, X_{2}^{I}\right) .
$$


(b) If $\left(X_{1}, X_{2}\right) \stackrel{\text { uo-disp }}{\lessgtr}\left(X_{1}^{I}, X_{2}^{I}\right)$ and $X_{i}$ is IFR for $i=1,2$, then $X_{i}$ is RTI in $X_{j}$, $i \neq j, i, j=1,2$.

ProOF:

(a) Note that $\operatorname{RTI}\left(X_{i} \mid X_{j}\right)$ if and only if, for all $u \geq 0$,

$$
\left\{X_{i} \mid X_{j}>F_{j}^{-1}(u)\right\} \geq_{\mathrm{hr}} X_{i}
$$

It follows from Theorem 3.1(a) that if, in addition, $X_{i}$ is DFR, then

$$
\left\{X_{i} \mid X_{j}>F_{j}^{-1}(u)\right\} \geq_{\mathrm{disp}} X_{i} \stackrel{\text { st }}{=} X_{i}^{I}
$$

Since this holds for $i, j=1,2$, the required result follows.

(b) $\left(X_{1}, X_{2}\right) \stackrel{\text { uo-disp }}{\lessgtr}\left(X_{1}^{I}, X_{2}^{I}\right)$ implies for all $u \geq 0$,

$$
\left\{X_{i} \mid X_{j}>F_{j}^{-1}(u)\right\} \geq_{\mathrm{disp}} X_{i}^{I} \stackrel{\text { st }}{=} X_{i}, \quad i \neq j, i, j=1,2
$$

This together with the assumption that $X_{1}$ and $X_{2}$ are IFR implies (3.2) by Theorem 3.1(b). This proves that $\operatorname{RTI}\left(X_{i} \mid X_{j}\right), i \neq j, i, j=1,2$.

Theorem 3.3: Let $\mathbf{X}$ and $\mathbf{Y}$ be two n-dimensional random vectors satisfying Assumption $A$ and such that $X_{i} \stackrel{\text { st }}{=} Y_{i}, i=1, \ldots, n$. Then $\mathbf{X} \stackrel{\text { uo-disp }}{\lessgtr} \mathbf{Y}$ implies that

$$
C_{i, j}^{X} \leq C_{i, j}^{Y} \quad \text { for } i, j=1, \ldots, n, i \neq j,
$$

where $C_{i, j}^{X}\left(C_{i, j}^{Y}\right)$ denotes the copula of $\left(X_{i}, X_{j}\right)\left(\left(Y_{i}, Y_{j}\right)\right)$.

PROOF:

$$
\mathbf{X} \stackrel{\text { uo-disp }}{\lessgtr} \mathbf{Y} \Rightarrow\left(X_{i}, X_{j}\right) \stackrel{\text { uo-disp }}{\lessgtr}\left(Y_{i}, Y_{j}\right), \quad i \neq j, i, j \in\{1, \ldots, n\},
$$

from which it follows that

$$
\left\{X_{i} \mid X_{j}>F_{j}^{-1}\left(u_{j}\right)\right\} \leq_{\text {disp }}\left\{Y_{i} \mid Y_{j}>G_{j}^{-1}\left(u_{j}\right)\right\}, \quad u_{j} \in[0,1]
$$

and which, in turn, implies

$$
\left\{X_{i} \mid X_{j}>F_{j}^{-1}\left(u_{j}\right)\right\} \leq_{\mathrm{st}}\left\{Y_{i} \mid Y_{j}>G_{j}^{-1}\left(u_{j}\right)\right\}, \quad u_{j} \in[0,1]
$$

under Assumption A since dispersive ordering implies stochastic ordering when the random variables have a finite common left end point of their supports. If we denote by $\bar{F}_{i, j}\left(\bar{G}_{i, j}\right)$ the joint survival function of $\left(X_{i}, X_{j}\right)\left(\left(Y_{i}, Y_{j}\right)\right)$, then (3.6) can be written as 


$$
\begin{aligned}
& \bar{F}_{i, j}\left(x, F_{j}^{-1}\left(u_{j}\right)\right) \leq \bar{G}_{i . j}\left(x, G_{j}^{-1}\left(u_{j}\right)\right) \quad \text { for } x \geq 0, u_{j} \in[0,1] \\
& \Leftrightarrow \bar{F}_{i, j}\left(F^{-1}{ }_{i}\left(u_{i}\right), F_{j}^{-1}\left(u_{j}\right)\right) \leq \bar{G}_{i, j}\left(F^{-1}{ }_{i}\left(u_{i}\right), G_{j}^{-1}\left(u_{j}\right)\right) \text { for all } u_{i}, u_{j} \in[0,1] \\
& \Leftrightarrow \bar{F}_{i, j}\left(F^{-1}{ }_{i}\left(u_{i}\right), F_{j}^{-1}\left(u_{j}\right)\right) \leq \bar{G}_{i, j}\left(G^{-1}{ }_{i}\left(u_{i}\right), G_{j}^{-1}\left(u_{j}\right)\right) \quad \text { for all } u_{i}, u_{j} \in[0,1] \\
& \text { ( since } X_{i} \stackrel{\text { st }}{=} Y_{i}, i=1, \ldots, n \text { ) } \\
& \Leftrightarrow \bar{C}_{i, j}^{X}\left(u_{i}, u_{j}\right) \leq \bar{C}_{i, j}^{Y}\left(u_{i}, u_{j}\right) \quad \text { for all } u_{i}, u_{j} \in[0,1] \\
& \Leftrightarrow C_{i, j}^{X}\left(u_{i}, u_{j}\right) \leq C_{i, j}^{Y}\left(u_{i}, u_{j}\right) \quad \text { for all } u_{i}, u_{j} \in[0,1] \text {, }
\end{aligned}
$$

where $\bar{C}(u, v)=1-u-v+C(u \cdot v)$.

If (3.4) holds and the margins of $\left(X_{i}, X_{j}\right)$ and $\left(Y_{i}, Y_{j}\right)$ are equal, then we say that $\left(Y_{i}, Y_{j}\right)$ is more PQD than $\left(X_{i}, X_{j}\right)$ (cf. [10, p. 36]). Note that $C_{i, j}^{X}\left(u_{i}, u_{j}\right) \equiv u_{i} u_{j}$ in the case $X_{i}$ and $X_{j}$ are independent and $C_{i, j}^{X}\left(u_{i}, u_{j}\right) \geq u_{i} u_{j}$ for all $u_{i}, u_{j} \in[0,1]$ in the case $X_{i}$ and $X_{j}$ are PQD. Thus, according to Theorem 3.3, if Assumption A holds and if $\mathbf{X}$ and $\mathbf{Y}$ have the same margins and $\mathbf{X} \stackrel{\text { uo-disp }}{\lessgtr} \mathbf{Y}$, then the $Y_{i}$ 's are more dependent than the $X_{i}$ 's according to PQD ordering. We obtain the following result as a special case.

Corollary 3.1: Let $\mathbf{X}=\left(X_{1}, X_{2}\right)$ be a bivariate random vector such that the left end point of the support of $\left\{X_{i} \mid X_{j}>F_{j}^{-1}(u)\right\}$ is finite and independent of $u \in[0,1]$, for $i \neq j$ and $i, j=1,2$. Let $\mathbf{X}^{\mathbf{I}}=\left(X_{1}^{I}, X_{2}^{I}\right)$ be a random vector of independent random variables such that $X_{i} \stackrel{\text { st }}{=} X_{i}^{I}, i=1,2$. Then

$$
\left(X_{1}, X_{2}\right) \stackrel{\text { uo-disp }}{\lessgtr}\left(X_{1}^{I}, X_{2}^{I}\right) \Rightarrow \mathbf{X} \text { is } P Q D .
$$

Contrast this result with Theorem 3.2(b), which is a stronger one since RTI implies PQD. However, here no assumption on the monotonicity of the hazard rates is made in the second case.

Remark 3.1: Assumption A is very crucial for Theorem 3.3 and Corollary 3.1 to hold. As a counterexample, let $Y_{1}$ and $Y_{2}$ be two independent $U(0,1)$ random variables. Let $X_{1}=X_{2}$ be uniformly distributed over $(0,1)$ also. Note that $X_{1}$ and $X_{2}$ are strongly positively dependent, as they satisfy the Frechet upper bound. Let us compare $\left(X_{1}, X_{2}\right)$ with $\left(Y_{1}, Y_{2}\right)$ according to upper orthant dispersive ordering. The relevant conditional distributions to compare are

$$
\left[X_{1} \mid X_{1}>u\right] \text { and }\left[Y_{1} \mid Y_{2}>u\right], \quad 0<u<1
$$

The left-hand conditional distribution is $U(u, 1)$ and the right-hand conditional distribution is $U(0,1)$. Hence, $\left(X_{1}, X_{2}\right) \stackrel{\text { uo-disp }}{\lessgtr}\left(Y_{1}, Y_{2}\right)$, but $C_{1,2}^{X} \geq C_{1,2}^{Y}$, contradictory to (3.4). The reason for this contradiction is that unless Assumption A is satisfied, dispersive ordering may not imply stochastic ordering. 
Theorem 3.4: Let $\mathbf{X}$ and $\mathbf{Y}$ be two n-dimensional random vectors satisfying Assumption $A$ and such that $\mathbf{X} \stackrel{\text { uo-disp }}{\lessgtr} \mathbf{Y}$. Then

$$
u v \leq C_{i, j}^{X} \leq C_{i, j}^{Y}, \quad i, j=1, \ldots, n, i \neq j,
$$

implies

$$
\operatorname{Cov}\left(h_{1}\left(X_{i}\right), h_{2}\left(X_{j}\right)\right) \leq \operatorname{Cov}\left(h_{1}\left(Y_{i}\right), h_{2}\left(Y_{j}\right)\right)
$$

for all increasing convex functions $h_{1}$ and $h_{2}$ for which the above covariances exist.

Proof: Without loss of generality, let $i=1$ and $j=2$. The survival functions of $\left(h_{1}\left(X_{1}\right), h_{2}\left(X_{2}\right)\right), h_{1}\left(X_{1}\right)$, and $h_{2}\left(X_{2}\right)$ are, respectively, $\bar{H}\left(x_{1}, x_{2}\right)=\bar{F}\left(h_{1}^{-1}\left(x_{1}\right)\right.$, $\left.h_{2}^{-1}\left(x_{2}\right)\right), \bar{H}_{1}\left(x_{1}\right)=\bar{F}_{1}\left(h_{1}^{-1}\left(x_{1}\right)\right)$, and $\bar{H}_{2}\left(x_{2}\right)=\bar{F}_{2}\left(h_{2}^{-1}\left(x_{2}\right)\right)$. Similarly, the survival functions of $\left(h_{1}\left(Y_{1}\right), h_{2}\left(Y_{2}\right)\right), h_{1}\left(Y_{1}\right)$, and $h_{2}\left(Y_{2}\right)$ are, respectively, $\bar{K}\left(x_{1}, x_{2}\right)=$ $\bar{G}\left(h_{1}^{-1}\left(x_{1}\right), h_{2}^{-1}\left(x_{2}\right)\right), \bar{K}_{1}\left(x_{1}\right)=\bar{G}_{1}\left(h_{1}^{-1}\left(x_{1}\right)\right)$, and $\bar{K}_{2}\left(x_{2}\right)=\bar{G}_{2}\left(h_{2}^{-1}\left(x_{2}\right)\right)$. Covariance between $h_{1}\left(X_{1}\right)$ and $h_{2}\left(X_{2}\right)$, if it exists, can be expressed as

$$
\begin{aligned}
\operatorname{Cov}\left(h_{1}\left(X_{1}\right), h_{2}\left(X_{2}\right)\right)= & \iint\left(\bar{H}\left(x_{1}, x_{2}\right)-\bar{H}_{1}\left(x_{1}\right) \bar{H}_{2}\left(x_{2}\right)\right) d x_{1} d x_{2} \\
= & \iint\left(\bar{F}\left(h_{1}^{-1}\left(x_{1}\right), h_{2}^{-1}\left(x_{2}\right)\right)\right. \\
& \left.-\bar{F}_{1}\left(h_{1}^{-1}\left(x_{1}\right)\right) \bar{F}_{2}\left(h_{2}^{-1}\left(x_{2}\right)\right)\right) d x_{1} d x_{2} \\
= & \iint\left(\bar{F}\left(F_{1}^{-1}(u), F_{2}^{-1}(v)\right)-(1-u)(1-v)\right) \\
& \times\left(\frac{h_{1}^{\prime}\left(F_{1}^{-1}(u)\right)}{f_{1}\left(F_{1}^{-1}(u)\right)}\right)\left(\frac{h_{2}^{\prime}\left(F_{2}^{-1}(v)\right)}{f_{2}\left(F_{2}^{-1}(v)\right)}\right) d u d v \\
= & \int_{0}^{1} \int_{0}^{1}\left(\bar{C}_{1,2}^{\mathbf{x}}(u, v)-(1-u)(1-v)\right) \\
& \times\left(\frac{h_{1}^{\prime}\left(F_{1}^{-1}(u)\right)}{f_{1}\left(F_{1}^{-1}(u)\right)}\right)\left(\frac{h_{2}^{\prime}\left(F_{2}^{-1}(v)\right)}{f_{2}\left(F_{2}^{-1}(v)\right)}\right) d u d v,
\end{aligned}
$$

where $h_{1}^{-1}\left(x_{1}\right)=F_{1}^{-1}(u)$ and $h_{2}^{-1}\left(x_{2}\right)=F_{2}^{-1}(v)$. The assumption $\mathbf{X} \stackrel{\text { uo-disp }}{\lessgtr} \mathbf{Y}$ implies that $X_{i} \leq_{\text {disp }} Y_{i}$, from which it follows that $f_{i}\left(F_{i}^{-1}(u)\right) \geq g_{i}\left(G_{i}^{-1}(u)\right)$ and $F_{i}^{-1}(u) \leq G_{i}^{-1}(u), i=1,2$, under Assumption A. Now $h_{i}^{\prime}(x)$ is increasing in $x$ since $h(x)$ is convex. Combining these facts, the required result follows from (3.8).

Theorem 3.5: Let $\mathbf{X}$ and $\mathbf{Y}$ be two $n$-dimensional random vectors satisfying the Assumption $A$ and let $\phi_{1}, \ldots, \phi_{n}$ be increasing convex functions on $\mathbb{R}^{+}$. Then

$$
\mathbf{X} \stackrel{\text { uo-disp }}{\lessgtr} \mathbf{Y} \Rightarrow\left(\phi_{1}\left(X_{1}\right), \ldots, \phi_{n}\left(X_{n}\right)\right) \stackrel{\text { uo-disp }}{\lessgtr}\left(\phi_{1}\left(Y_{1}\right), \ldots, \phi_{n}\left(Y_{n}\right)\right) \text {. }
$$


Proof: Note that the cdf of $\phi_{j}\left(X_{j}\right)$ is $F_{\phi_{j}}(x)=F_{j}\left(\phi_{j}^{-1}(x)\right)$, with its inverse as $F_{\phi_{j}}^{-1}(u)=\phi_{j}\left(F_{j}^{-1}(u)\right)$. We have to prove that for $i=1, \ldots, n, u_{j} \in(0,1), j=1, \ldots, n$, $j \neq i$

$$
\left(\phi_{i}\left(X_{i}\right) \mid \bigcap_{j \neq i}\left\{\phi_{j}\left(X_{j}\right)>F_{\phi_{j}}^{-1}\left(u_{j}\right)\right\} \leq_{\operatorname{disp}}\left(\phi_{i}\left(Y_{i}\right) \mid \bigcap_{j \neq i}\left\{\phi_{j}\left(Y_{j}\right)>G_{\phi_{j}}^{-1}\left(u_{j}\right)\right\}\right) ;\right.
$$

that is,

$$
\begin{aligned}
& \left(\phi_{i}\left(X_{i}\right) \mid \bigcap_{j \neq i}\left\{\phi_{j}\left(X_{j}\right)>\phi_{j}\left(F_{j}^{-1}\left(u_{j}\right)\right)\right\}\right) \\
& \quad \leq_{\operatorname{disp}}\left(\phi_{i}\left(Y_{i}\right) \mid \bigcap_{j \neq i}\left\{\phi_{j}\left(Y_{j}\right)>\phi_{j}\left(G_{j}^{-1}\left(u_{j}\right)\right)\right\}\right),
\end{aligned}
$$

which is equivalent to

$$
\left(\phi_{i}\left(X_{i}\right) \mid \bigcap_{j \neq i}\left\{X_{j}>F_{j}^{-1}\left(u_{j}\right)\right\}\right) \leq_{\operatorname{disp}}\left(\phi_{i}\left(Y_{i}\right) \mid \bigcap_{j \neq i}\left\{Y_{j}>G_{j}^{-1}\left(u_{j}\right)\right\}\right) .
$$

Using Assumption A, for $i=1, \ldots, n, u_{j} \in(0,1), j=1, \ldots, n, j \neq i$,

$$
\left(X_{i} \mid \bigcap_{j \neq i}\left\{X_{j}>F_{j}^{-1}\left(u_{j}\right)\right\}\right) \leq_{\operatorname{disp}}\left(Y_{i} \mid \bigcap_{j \neq i}\left\{Y_{j}>G_{j}^{-1}\left(u_{j}\right)\right\}\right)
$$

implies

$$
\left(X_{i} \mid\left\{X_{j}>F_{j}^{-1}\left(u_{j}\right)\right\}\right) \leq_{\mathrm{st}}\left(Y_{i} \mid\left\{Y_{j}>G_{j}^{-1}\left(u_{j}\right)\right\}\right) .
$$

Now the required result follows from Theorem 2.2 of Rojo and He [18] since $\phi_{i}$ 's are increasing convex functions.

Hu et al. [9] gave the following definition of multivariate (weak) hazard rate ordering.

Definition 3.1: Let $\mathbf{X}$ and $\mathbf{Y}$ be $n$-dimensional random vectors with hazard gradients $r_{\mathbf{X}}$ and $r_{\mathbf{Y}}$, respectively. We say that $\mathbf{X}$ is smaller than $\mathbf{Y}$ according to weak hazard rate ordering (written as $\mathbf{X} \leq_{\text {whr }} \mathbf{Y}$ ) if

$$
\left(X_{i} \mid \bigcap_{j \neq i}\left\{X_{j}>x_{j}\right\}\right) \leq_{\mathrm{hr}}\left(Y_{i} \mid \bigcap_{j \neq i}\left\{Y_{j}>x_{j}\right\}\right)
$$

for $i=1,2, \ldots, n, \mathbf{x} \in \mathbb{R}^{n}$; that is, if

$$
r_{\mathbf{X}}^{(i)}(\mathbf{x}) \geq r_{\mathbf{Y}}^{(i)}(\mathbf{x}), \quad i=1,2, \ldots, n, \mathbf{x} \in \mathbb{R} .
$$

In the next theorem we establish results analogous to Theorem 3.1 between upper orthant dispersive ordering and multivariate weak hazard rate ordering under Assumption A. 
TheOREm 3.6: Let $\mathbf{X}$ and $\mathbf{Y}$ be two n-dimensional random vectors satisfying Assumption A.

(a) If $\mathbf{Y} \leq_{\text {whr }} \mathbf{X}$ and either $r_{\mathbf{X}}^{(i)}(\mathbf{x})$ or $r_{\mathbf{Y}}^{(i)}(\mathbf{x})$ is decreasing in $\mathbf{x}$, for $i=1, \ldots, n$, then $\mathbf{Y} \stackrel{\text { uo-disp }}{\lessgtr} \mathbf{X}$.

(b) If $\mathbf{Y} \stackrel{\text { uo-disp }}{\lessgtr} \mathbf{X}$ and either $r_{\mathbf{X}}^{(i)}(\mathbf{x})$ or $r_{\mathbf{Y}}^{(i)}(\mathbf{x})$ is increasing in $\mathbf{x}$, for $i=1, \ldots, n$, then $\mathbf{Y} \leq_{\text {whr }} \mathbf{X}$.

ProOF:

(a) Under Assumption A, $\mathbf{Y} \leq_{\text {whr }} \mathbf{X}$ implies that $Y_{i} \leq_{\mathrm{st}} X_{i}, i=1, \ldots, n$, which is equivalent to $G_{i}^{-1}(u) \leq F_{i}^{-1}(u)$, for $u \in[0,1], i=1, \ldots, n$. Again, $\mathbf{Y} \leq_{\mathrm{whr}} \mathbf{X}$ implies that for $i=1, \ldots, n,\left(Y_{i} \mid \bigcap_{j \neq i}\left\{Y_{j}>x_{j}\right\}\right) \leq_{\mathrm{st}}\left(X_{i} \mid \cap_{j \neq i}\left\{X_{j}>x_{j}\right\}\right)$. Taking $x_{j}=F_{j}^{-1}\left(u_{j}\right), j=1, \ldots, n, j \neq i$, we find that this implies $y_{i}^{\prime}(\beta ; \mathbf{u}) \leq$ $x_{i}(\beta ; \mathbf{u})$, where $y_{i}^{\prime}(\beta ; \mathbf{u})$ is the $\beta$ th quantiles of the conditional distribution $\left(Y_{i} \mid \bigcap_{j \neq i}\left\{Y_{j}>F_{j}^{-1}\left(u_{j}\right)\right\}\right)$ and $x_{i}(\beta ; \mathbf{u})$ is as defined earlier. On the other hand, $r_{\mathbf{Y}}^{(i)}(\mathbf{x})$ decreasing in $\mathbf{x}$ implies that

$$
\left(Y_{i} \mid \bigcap_{j \neq i}\left\{Y_{j}>x_{j}\right\}\right) \mathbf{s}_{\mathrm{st}}\left(Y_{i} \mid \bigcap_{j \neq i}\left\{Y_{j}>x_{j}^{\prime}\right\}\right),
$$

for $x_{j} \leq x_{j}^{\prime}, j=1 \ldots, n, j \neq i$. This, along with $G_{i}^{-1}\left(u_{i}\right) \leq F_{i}^{-1}\left(u_{i}\right)$, implies that

$$
y_{i}(\beta ; \mathbf{u}) \leq y_{i}^{\prime}(\beta ; \mathbf{u}) \leq x_{i}(\beta ; \mathbf{u}), \quad i=1, \ldots, n .
$$

Using these observations, we obtain

$$
\begin{aligned}
& r_{\mathbf{X}}^{(i)}\left(F_{1}^{-1}\left(u_{1}\right), \ldots, F_{i-1}^{-1}\left(u_{i-1}\right), x_{i}(\beta ; \mathbf{u}), \ldots, F_{n}^{-1}\left(u_{n}\right)\right) \\
& \quad \leq r_{\mathbf{Y}}^{(i)}\left(F_{1}^{-1}\left(u_{1}\right), \ldots, F_{i-1}^{-1}\left(u_{i-1}\right), x_{i}(\beta ; \mathbf{u}), \ldots, F_{n}^{-1}\left(u_{n}\right)\right),
\end{aligned}
$$

since $\mathbf{Y} \leq_{\text {whr }} \mathbf{X}$. The right-hand side of this inequality is less than or equal to

$$
r_{\mathbf{Y}}^{(i)}\left(G_{1}^{-1}\left(u_{1}\right), \ldots, G_{i-1}^{-1}\left(u_{i-1}\right), y_{i}(\beta ; \mathbf{u}), \ldots, G_{n}^{-1}\left(u_{n}\right)\right)
$$

since $r_{\mathbf{Y}}^{(i)}(\mathbf{x})$ is decreasing in $\mathbf{x}$. This completes the proof of (a).

(b) From the assumption $\mathbf{Y} \stackrel{\text { uo-disp }}{\lessgtr} \mathbf{X}$, it follows that

$$
\begin{aligned}
& r_{\mathbf{X}}^{(i)}\left(F_{1}^{-1}\left(u_{1}\right), \ldots, F_{i-1}^{-1}\left(u_{i-1}\right), x_{i}(\beta ; \mathbf{u}), \ldots, F_{n}^{-1}\left(u_{n}\right)\right) \\
& \quad \leq r_{\mathbf{Y}}^{(i)}\left(G_{1}^{-1}\left(u_{1}\right), \ldots, G_{i-1}^{-1}\left(u_{i-1}\right), y_{i}(\beta ; \mathbf{u}), \ldots, G_{n}^{-1}\left(u_{n}\right)\right)
\end{aligned}
$$


and

$$
\left(X_{i} \mid \bigcap_{j \neq i}\left\{X_{j}>F_{j}^{-1}\left(u_{j}\right)\right\}\right) \geq_{\mathrm{st}}\left(Y_{i} \mid \bigcap_{j \neq i}\left\{Y_{j}>G_{j}^{-1}\left(u_{j}\right)\right\}\right),
$$

which implies that $x_{i}(\beta ; \mathbf{u}) \geq y_{i}(\beta ; \mathbf{u})$ and $F_{j}^{-1}\left(u_{j}\right) \geq G_{j}^{-1}\left(u_{j}\right), j=1, \ldots, n$, $j \neq i$. Using these facts and the assumption that $r_{\mathbf{Y}}^{(i)}(\mathbf{x})$ is increasing in $\mathbf{x}$, it follows that, for $i=1, \ldots, n$, the right-hand side of (3.11) is less than or equal to

$$
r_{\mathbf{Y}}^{(i)}\left(F_{1}^{-1}\left(u_{1}\right), \ldots, F_{i-1}^{-1}\left(u_{i-1}\right), x_{i}(\beta ; \mathbf{u}), \ldots, F_{n}^{-1}\left(u_{n}\right)\right)=r_{\mathbf{Y}}^{(i)}(\mathbf{x})
$$

that is, we have shown that for $i=1, \ldots, n$,

$$
r_{\mathbf{X}}^{(i)}(\mathbf{x}) \leq r_{\mathbf{Y}}^{(i)}(\mathbf{x})
$$

and hence the required result.

Remark 3.2: If $r_{\mathbf{X}}^{(i)}\left(x_{1}, \ldots, x_{i}, \ldots, x_{n}\right)$ increases in $x_{i}$ for $i=1, \ldots, n$, then we say that the random vector $\mathbf{X}$ has a multivariate increasing hazard rate distribution (cf. Johnson and Kotz [11]). The condition $r_{\mathbf{X}}^{(i)}\left(x_{1}, \ldots, x_{i}, \ldots, x_{n}\right)$ increasing in $x_{j}, j=$ $1, \ldots, n, j \neq i, i=1, \ldots, n$ describes a condition of positive dependence that is equivalent to saying that the random vector $\mathbf{X}$ has RCSI; that is,

$$
P\left[X_{1}>x_{1}, \ldots, X_{n}>x_{n} \mid X_{1}>x_{1}^{\prime}, \ldots, X_{n}>x_{n}^{\prime}\right]
$$

increases in $x_{i}^{\prime}, i=1, \ldots, n$.

We will now study some preservation properties of the upper orthant dispersive order under random compositions. Such results are often referred to as preservations under "random mapping" (see Shaked and Wong [22]), or preservations of "stochastic convexity" (see Shaked and Shanthikumar [20, Chap. 6] and Denuit, Lefèvre, and Utev [6], and references therein).

Let $\left\{\bar{F}_{\theta}, \theta \in \mathcal{X}\right\}$ be a family of $n$-dimensional survival functions, where $\mathcal{X}$ is a subset of the real line. Let $\boldsymbol{X}(\theta)$ denote a random vector with survival function $\bar{F}_{\theta}$. For any random variable $\Theta$ with support in $\mathcal{X}$ and with distribution function $H$, let us denote by $\boldsymbol{X}(\Theta)$ a random vector with survival function $\bar{G}$ given by

$$
\bar{G}(\boldsymbol{x})=\int_{\mathcal{X}} \bar{F}_{\theta}(\boldsymbol{x}) d H(\theta), \quad \boldsymbol{x} \in \mathbb{R}^{n} .
$$

THeORem 3.7: Consider a family of $n$-dimensional survival functions $\left\{\bar{F}_{\theta}, \theta \in \mathcal{X}\right\}$ as above. Let $\Theta_{1}$ and $\Theta_{2}$ be two random variables with supports in $\mathcal{X}$ and distribution functions $H_{1}$ and $H_{2}$, respectively. Let $\boldsymbol{Y}_{1}$ and $\boldsymbol{Y}_{2}$ be two random vectors such that $\boldsymbol{Y}_{i}={ }_{\mathrm{st}} \boldsymbol{X}\left(\Theta_{i}\right), i=1,2$; that is, suppose that the survival function of $\boldsymbol{Y}_{i}$ is given by

$$
\bar{G}_{i}(\boldsymbol{x})=\int_{\mathcal{X}} \bar{F}_{\theta}(\boldsymbol{x}) d H_{i}(\theta), \quad \boldsymbol{x} \in \mathbb{R}, i=1,2 .
$$


If

(a)

$$
\boldsymbol{X}(\theta) \leq_{\mathrm{whr}} \boldsymbol{X}\left(\theta^{\prime}\right) \quad \text { whenever } \theta \leq \theta^{\prime},
$$

(b) $\Theta_{1}$ and $\Theta_{2}$ are ordered in the univariate hazard rate order; that is,

$$
\Theta_{1} \leq_{\mathrm{hr}} \Theta_{2},
$$

(c) $r_{\mathbf{X}(\theta)}^{(i)}\left(x_{1}, \ldots, x_{n}\right)$ is decreasing in $x_{j}, j=1, \ldots, n, i=1, \ldots, n$, then

$$
\mathbf{Y}_{\mathbf{1}} \stackrel{\text { uo-disp }}{\lessgtr} \mathbf{Y}_{\mathbf{2}} \text {. }
$$

Proof: Hu et al. [9] proved that assumptions (a) and (b) imply that $\boldsymbol{Y}_{1} \leq_{\text {whr }} \boldsymbol{Y}_{2}$. Now, we show that for $i=1, \ldots, n, r_{\mathbf{Y}_{\mathbf{1}}}^{(i)}\left(x_{1}, \ldots, x_{n}\right)$ is decreasing in $x_{j}, j=1, \ldots, n$, then the required result will follow from Theorem 3.6(a). Assumption (a) is equivalent to $\bar{F}_{\theta}(\mathbf{x})$ being $T P_{2}$ (recall from Karlin [12] that a function $f: \mathbb{R}^{2} \rightarrow \mathbb{R}$ is said to be totally positive of order $2\left(T P_{2}\right)$ if $f\left(x_{1}, y_{1}\right) f\left(x_{2}, y_{2}\right) \geq f\left(x_{1}, y_{2}\right) f\left(x_{2}, y_{1}\right)$, for $x_{2} \geq x_{1}$ and $\left.y_{2} \geq y_{1}\right)$ in $\left(\theta, x_{j}\right), j=1, \ldots, n . r_{\mathbf{X}(\theta)}^{(i)}\left(x_{1}, \ldots, x_{n}\right)$ decreasing in $x_{j}, j=1, \ldots, n, j \neq i$ is equivalent to $\bar{F}_{\theta}\left(x_{1}, \ldots, x_{n}\right)$ being $T P_{2}$ in $\left(x_{i}, x_{j}\right), i, j=$ $1, \ldots, n, j \neq i$. Using these observations, it follows that $\bar{G}_{1}(\mathbf{x})$ is $T P_{2}$ in $\left(x_{i}, x_{j}\right)$, $i, j=1, \ldots, n$ (cf. Karlin [12]), which is equivalent to $r_{\mathbf{Y}_{1}}^{(i)}\left(x_{1}, \ldots, x_{n}\right)$ decreasing in $x_{j}, j=1, \ldots, n, j \neq i$. It is worth noting that $r_{\mathbf{X}(\theta)}^{(i)}\left(x_{1}, \ldots, x_{n}\right)$ decreasing in $x_{i}$ is equivalent to the fact that $\left\{X_{i} \mid \cap_{j \neq i}\left\{X_{j}>x_{j}\right\}\right\}$ is a DFR random variable, $i=$ $1, \ldots, n$. Now, $\bar{G}_{1}(\mathbf{x})$ can be written as

$\bar{G}_{1}(\mathbf{x})=\int P_{\theta}\left(X_{i}>x_{i} \mid \bigcap_{j \neq i}\left\{X_{j}>x_{j}\right\}\right) P_{\theta}\left(\bigcap_{j \neq i}\left\{X_{j}>x_{j}\right\}, j=1, \ldots, n, j \neq i\right) d H_{1}(\theta) ;$

that is, $\left(Y_{1}, \ldots, Y_{n}\right)$ is a sort of mixture of $D F R$ random variables; therefore, $-\partial \log \bar{G}_{j}(\mathbf{x}) / \partial x_{i}$ is decreasing in $x_{i}$, which is equivalent to $r_{\mathbf{Y}_{1}}^{(i)}\left(x_{1}, \ldots, x_{n}\right)$ decreasing in $x_{i}$. This completes the proof.

\section{EXAMPLES AND APPLICATIONS}

Example 4.1 (Multivariate Pareto Distributions): For $a>0$, let $\mathbf{X}_{a}=\left(X_{a, 1}, \ldots, X_{a, n}\right)$ have the survival function $\bar{F}_{a}$ given by

$$
\bar{F}_{a}\left(x_{1}, \ldots, x_{n}\right)=\left(\sum_{i=1}^{n} x_{i}-n+1\right)^{-a}, \quad x_{i}>1, i=1,2, \ldots, n ;
$$

see, for example, Kotz, Balakrishnan, and Johnson [14, p. 600]. The corresponding density function is given by

$$
\begin{gathered}
f_{a}\left(x_{1}, \ldots, x_{n}\right)=a(a+1) \ldots(a+n-1)\left(\sum_{i=1}^{n} x_{i}-n+1\right)^{-a-n}, \\
x_{i}>1, i=1,2, \ldots, n .
\end{gathered}
$$


Hu et al. [9] showed that $\mathbf{X}_{a_{1}} \leq_{\text {whr }} \mathbf{X}_{a_{2}}$ whenever $a_{1} \geq a_{2}$. On the other hand, $r_{\mathbf{X}}^{(i)}(\mathbf{x})=a /\left(\sum_{j=1}^{n} x_{j}-n+1\right)$, is decreasing in $x_{j}, j=1, \ldots, n$. Then from Theorem 3.6(a) it follows that $\mathbf{X}_{a_{1}} \stackrel{\text { uo-disp }}{\lessgtr} \mathbf{X}_{a_{2}}$ whenever $a_{1} \geq a_{2}$.

Example 4.2 (Bivariate Farlie-Gumbel-Morgenstern Distributions): For $\alpha \in$ $(-1,1)$, let $\mathbf{X}_{\alpha}=\left(X_{\alpha, 1}, X_{\alpha, 2}\right)$ have the survival function $\bar{F}_{\alpha}$ given by

$$
\bar{F}_{\alpha}\left(x_{1}, x_{2}\right)=\bar{F}_{1}\left(x_{1}\right) \bar{F}_{2}\left(x_{2}\right)\left[1+\alpha\left(1-\bar{F}_{1}\left(x_{1}\right)\right)\left(1-\bar{F}_{2}\left(x_{1}\right)\right)\right]
$$

and $\mathbf{Y}_{\alpha}=\left(Y_{\alpha, 1}, Y_{\alpha, 2}\right)$ have the survival function $\bar{G}_{\alpha}$ given by

$$
\bar{G}_{\alpha}\left(x_{1}, x_{2}\right)=\bar{G}_{1}\left(x_{1}\right) \bar{G}_{2}\left(x_{2}\right)\left[1+\alpha\left(1-\bar{G}_{1}\left(x_{1}\right)\right)\left(1-\bar{G}_{2}\left(x_{1}\right)\right)\right],
$$

where $\bar{F}_{1}, \bar{F}_{2}, \bar{G}_{1}$, and $\bar{G}_{2}$ are arbitrary univariate survival functions (which happen to be the marginal survival functions of $X_{\alpha, 1}, X_{\alpha, 2}, Y_{\alpha, 1}$, and $Y_{\alpha, 2}$, respectively, independently of $\alpha$ ). Assume that $X_{\alpha, i} \leq_{\text {disp }} Y_{\alpha, i}, i=1,2$. It is easy to see that $\bar{C}^{\mathbf{x}_{\alpha}}(u, v)=\bar{C}^{\mathbf{Y}_{\alpha}}(u, v)$. Then, from Theorem 2.1, it follows that $\mathbf{X}_{\alpha} \stackrel{\text { uo-disp }}{\lessgtr} \mathbf{Y}_{\alpha}$.

Example 4.3 (Multivariate Gumbel Exponential Distributions): For positive parameters $\boldsymbol{\lambda}=\left\{\lambda_{I}: I \subseteq\{1,2, \ldots, n\}, I \neq \varnothing\right\}$, let $\boldsymbol{X}_{\boldsymbol{\lambda}}=\left(X_{1}, X_{2}, \ldots, X_{n}\right)$ have the survival function $\bar{F}_{\lambda}$ given by

$$
\bar{F}_{\lambda}\left(x_{1}, x_{2}, \ldots, x_{n}\right)=\exp \left\{-\sum_{I} \lambda_{I} \prod_{i \in I} x_{i}\right\}, \quad\left(x_{1}, x_{2}, \ldots, x_{n}\right) \geq(0,0, \ldots, 0) ;
$$

see Kotz et al. [14, p. 406]. For another set of positive parameters $\lambda^{*}=\left\{\lambda_{I}^{*}: I \subseteq\right.$ $\{1,2, \ldots, n\}, I \neq \varnothing\}$, let $\boldsymbol{Y}_{\boldsymbol{\lambda}^{*}}=\left(Y_{1}, Y_{2}, \ldots, Y_{n}\right)$ have the survival function $\bar{G}_{\boldsymbol{\lambda}^{*}}$. Let $X_{i}={ }_{\text {st }} Y_{i}, i=1, \ldots, n$; that is, $\lambda_{i}=\lambda_{i}^{*}$. We show that if $\boldsymbol{\lambda} \geq \boldsymbol{\lambda}^{*}$, then for $i=1, \ldots, n$,

$$
\left(X_{i} \mid \bigcap_{j \neq i}\left\{X_{j}>F_{j}^{-1}\left(u_{j}\right)\right\}\right) \leq_{\operatorname{disp}}\left(Y_{i} \mid \bigcap_{j \neq i}\left\{Y_{j}>G_{j}^{-1}\left(u_{j}\right)\right\}\right), \quad u_{j} \in[0,1] .
$$

Since $X_{i}={ }_{\mathrm{st}} Y_{i}, i=1, \ldots, n,(4.1)$ is equivalent to

$$
\left(X_{i} \mid \bigcap_{j \neq i}\left\{X_{j}>x_{j}\right\}\right) \leq_{\mathrm{disp}}\left(Y_{i} \mid \bigcap_{j \neq i}\left\{Y_{j}>x_{j}\right\}\right), \quad i=1, \ldots, n .
$$

Let $\mathbf{x}_{\mathbf{i}}=\left(x_{1}, \ldots, x_{i-1}, x_{i+1}, \ldots, n\right)$. The survival function of $\left(X_{i} \mid \bigcap_{j \neq i}\left\{X_{j}>x_{j}\right\}\right)$, denoted by $\bar{F}_{i}\left(x_{i} ; \mathbf{x}_{\mathbf{i}}\right)$, is

$\bar{F}_{i}\left(x_{i} ; \mathbf{x}_{\mathbf{i}}\right)=\exp \left\{-x_{i}\left\{\lambda_{i}+\sum_{j} \lambda_{i j} x_{j}+\sum_{j \neq k} \lambda_{i j k} x_{j} x_{k}+\cdots+\lambda_{12 \ldots n} \prod_{j \neq i} x_{j}\right\}\right\}$.

Similarly, the survival function of $\left\{Y_{i} \mid Y_{j}>x_{j}, \quad j \neq i\right\}$, denoted by $\bar{G}_{i}\left(x_{i} ; \mathbf{x}_{\mathbf{i}}\right)$, is

$$
\bar{G}_{i}\left(x_{i} ; \mathbf{x}_{\mathbf{i}}\right)=\exp \left\{-x_{i}\left\{\lambda_{i}+\sum_{j} \lambda_{i j}^{*} x_{j}+\sum_{j \neq k} \lambda_{i j k}^{*} x_{j} x_{k}+\cdots+\lambda_{12 \ldots n}^{*} \prod_{j \neq i} x_{j}\right\}\right\} .
$$


Now, the ratio

$$
\frac{\bar{G}_{i}\left(x_{i} ; \mathbf{x}_{\mathbf{i}}\right)}{\bar{F}_{i}\left(x_{i} ; \mathbf{x}_{\mathbf{i}}\right)}
$$

is increasing in $x_{i}$; that is,

$$
\left(X_{i} \mid \bigcap_{j \neq i}\left\{X_{j}>x_{j}\right\}\right) \leq_{\mathrm{hr}}\left(Y_{i} \mid \bigcap_{j \neq i}\left\{Y_{j},>x_{j}\right\}\right), \quad i=1, \ldots, n .
$$

On the other hand, the random variable $\left\{X_{i} \mid X_{j}>x_{j}, j \neq i\right\}$ has an exponential distribution that is DFR. Combining this observation with (4.6), it follows from Theorem 3.1(a) that (4.2) holds. Now, applying Theorem 3.3 to this example, we get that $C_{i, j}^{\lambda}$ is decreasing in $\lambda$, where $C_{i, j}^{\lambda}$ denotes the copula of $\left(X_{i}, X_{j}\right)$

Application 4.1 (Order Statistics): Let $X_{1}, \ldots, X_{n}\left(Y_{1}, \ldots, Y_{n}\right)$ be a random sample from a univariate distribution with strictly increasing distribution function $F(G)$. Bartoszewicz [3] has shown that $F \leq_{\text {disp }} G$ implies $X_{i: n} \leq_{\text {disp }} Y_{i: n}, i=1, \ldots, n$, where $X_{i: n}\left(Y_{i: n}\right)$ is the $i$ th-order statistic of the $X$ sample ( $Y$ sample). We will strengthen this result to prove that $F \leq_{\text {disp }} G$ implies

$$
\left(X_{1: n}, \ldots, X_{n: n}\right) \stackrel{\text { uo-disp }}{\lessgtr}\left(Y_{1: n}, \ldots, Y_{n: n}\right) \text {. }
$$

We first show that in the case of random samples from continuous distributions, the copulas of order statistics are independent of the parent distributions. Note that $Y_{i: n} \stackrel{\text { st }}{=} G^{-1} F\left(X_{i: n}\right), i=1, \ldots n$. Since the function $G^{-1} F$ is strictly increasing, it follows from Theorem 2.4.3 of Nelsen [17] that $C\left(X_{i: n}, X_{j: n}\right)=C\left(Y_{i: n}, Y_{j: n}\right)$, for $i, j=1, \ldots, n$. It now immediately follows from Theorem 2.1 that $F \leq_{\text {disp }} G$ implies (4.7).

Since the order statistics from a random sample are positively associated (cf. Boland, Hollander, Joag-Dev, and Kochar [4] $)$ and since $\left(X_{1: n}, \ldots, X_{n: n}\right)$ and $\left(Y_{1: n}, \ldots\right.$, $\left.Y_{n: n}\right)$ have the same copula, the conditions of Theorem 3.4 are satisfied. Hence, for $i, j \in\{1, \ldots, n\}$,

$$
\operatorname{Cov}\left(h_{1}\left(X_{i: n}\right), h_{2}\left(X_{j: n}\right)\right) \leq \operatorname{Cov}\left(h_{1}\left(Y_{i: n}\right), h_{2}\left(Y_{j: n}\right)\right)
$$

for all increasing convex functions $h_{1}$ and $h_{2}$ for which the above covariances exist. This result was originally proved by Bartoszewicz [2] using a different method. A similar result can be established for record values.

Application 4.2 (Record Values): Let $X_{1}, \ldots, X_{n}, \ldots\left(Y_{1}, \ldots, Y_{n}, \ldots\right)$ be a sequence of random variables from a univariate distribution $F(G)$. It is known that $F \leq_{\text {disp }} G$ implies $R_{m}^{X} \leq_{\text {disp }} R_{m}^{Y}$, where $R_{m}^{X}\left(R_{m}^{Y}\right)$ is the $m$ th record value of the $X$ sequence ( $Y$ sequence). We first show that in the case of random sequences from continuous distributions, the copulas of record values are independent of the parent distributions. Then it will immediately follow from Theorem 2.1 that $F \leq_{\text {disp }} G$ implies 


$$
\left(R_{m_{1}}^{X}, \ldots, R_{m_{n}}^{X}\right) \stackrel{\text { uo-disp }}{\lessgtr}\left(R_{m_{1}}^{Y}, \ldots, R_{m_{n}}^{Y}\right) .
$$

Let $M(x)=-\log \bar{F}(x)$; then the distribution function of $R_{m}^{X}$ can be expressed as $F_{R_{m}^{X}}(x)=G_{m}(M(x))$, where $G_{m}(x)$ is the distribution function of a Gamma random variable with scale parameter one and shape parameter $m$. Similarly, let $N(x)=$ $-\log \bar{G}(x)$; then the distribution function of $R_{m}^{Y}$ is $F_{R_{m}^{Y}}(x)=G_{m}(N(x))$. Now, both $M\left(X_{1}\right), \ldots, M\left(X_{n}\right), \ldots$ and $N\left(Y_{1}\right), \ldots, N\left(Y_{n}\right), \ldots$ are sequences of independent and identically distributed (i.i.d.) exponential random variables with mean one. Using this observation, it follows that

$$
\left(R_{m_{1}}^{X}, \ldots, R_{m_{n}}^{X}\right) \stackrel{\mathrm{st}}{=}\left(M^{-1}\left(R_{m_{1}}^{*}\right), \ldots, M^{-1}\left(R_{m_{n}}^{*}\right)\right)
$$

and

$$
\left(R_{m_{1}}^{Y}, \ldots, R_{m_{n}}^{Y}\right) \stackrel{\text { st }}{=}\left(N^{-1}\left(R_{m_{1}}^{*}\right), \ldots, N^{-1}\left(R_{m_{n}}^{*}\right)\right)
$$

where $R_{m}^{*}$ is the $m$ th record value of a sequence of i.i.d. exponential random variables with mean one.

$$
\begin{aligned}
C^{\mathbf{R}^{X}}\left(u_{1}, \ldots, u_{n}\right)= & F_{\mathbf{R}^{X}}\left(F_{R_{m_{1}}^{X}}^{-1}\left(u_{1}\right), \ldots, F_{R_{m_{n}}^{X}}^{-1}\left(u_{n}\right)\right) \\
= & P\left(R_{m_{1}}^{X} \leq F_{R_{m_{1}}^{X}}^{-1}\left(u_{1}\right), \ldots, R_{m_{n}}^{X} \leq F_{R_{m_{n}}^{X}}^{-1}\left(u_{n}\right)\right) \\
= & P\left(M^{-1}\left(R_{m_{1}}^{*}\right) \leq M^{-1} G_{m_{1}}^{-1}\left(u_{1}\right), \ldots, M^{-1}\left(R_{m_{n}}^{*}\right)\right. \\
& \left.\quad \leq M^{-1} G_{m_{n}}^{-1}\left(u_{n}\right)\right) \\
= & P\left(R_{m_{1}}^{*} \leq G_{m_{1}}^{-1}\left(u_{1}\right), \ldots, R_{m_{n}}^{*} \leq G_{m_{n}}^{-1}\left(u_{n}\right)\right) \\
= & P\left(N^{-1}\left(R_{m_{1}}^{*}\right) \leq N^{-1} G_{m_{1}}^{-1}\left(u_{1}\right), \ldots, N^{-1}\left(R_{m_{n}}^{*}\right) \leq N^{-1} G_{m_{n}}^{-1}\left(u_{n}\right)\right) \\
= & P\left(R_{m_{1}}^{Y} \leq F_{R_{m_{1}}}^{-1}\left(u_{1}\right), \ldots, R_{m_{n}}^{Y} \leq F_{R_{m_{n}}}^{-1}\left(u_{n}\right)\right) \\
= & C^{\mathbf{R}^{Y}}\left(u_{1}, \ldots, u_{n}\right) .
\end{aligned}
$$

This proves the desired result.

\section{Acknowledgments}

Research by the first author was partially supported by the Statistical Research Center, Tehran, Iran. The authors are grateful to the referees for their helpful comments and suggestions, which have greatly improved the presentation of the article.

\section{References}

1. Bagai, I. \& Kochar, S.C. (1986). On tail ordering and comparison of failure rates. Communications in Statistics-Theory and Methods 15: 1377-1388.

2. Bartoszewicz, J. (1985). Moment inequalities for order statistics from ordered families of distributions. Metrika 32: 383-389. 
3. Bartoszewicz, J. (1986). Dispersive ordering and the total time on test transformation. Statistics and Probability Letters 4: 285-288.

4. Boland, P.J., Hollander, M., Joag-Dev, K., \& Kochar, S. (1996). Bivariate dependence properties of order statistics. Journal of Multivariate Analysis 56: 75-89.

5. Deheuvels, P. (1978). Caractérisation compelet des lois extrêmes multivariées et de la convergence des types extrêmes. Publication du Institute Statistics du Universite de Paris 23: 1-37.

6. Denuit, M., Lefèvre, C., \& Utev, S. (1999). Generalized stochastic convexity and stochastic orderings of mixtures. Probability in the Engineering and Informational Sciences 13: 275-291.

7. Fernandez-Ponce, J.M. \& Suarez-Llorens, A. (2003). A multivariate dispersion ordering based on quantiles more widely separated. Journal of Multivariate Analysis 85: 40-53.

8. Giovagnoli, A. \& Wynn, H.P. (1995). Multivariate dispersion orderings. Statistics and Probability Letters 22: 325-332.

9. Hu, T., Khaledi, B.E., \& Shaked, M. (2002). Multivariate hazard rate orders. Journal of Multivariate Analysis 84: 173-189.

10. Joe, H. (1997). Multivariate models and dependence concepts. London: Chapman \& Hall.

11. Johnson, N.L. \& Kotz, S. (1975). A vector multivariate hazard rate. Journal of Multivariate Analysis 5: 53-66.

12. Karlin, S. (1968). Total positivity. Stanford, CA: Stanford University Press.

13. Kimeldrof, G. \& Sampson, A. (1975). Uniform representations of bivariate distributions with fixed marginals. Communications in Statistics-Theory and Methods 4: 617-627.

14. Kotz, S., Balakrishnan, N., \& Johnson, N.L. (2000). Continuous multivariate distributions, Vol. 1: Models and applications, 2nd ed., New York: Wiley.

15. Marshall, A.W. (1975). Some comments on the hazard gradient. Stochastic Processes and Their Applications 3: 293-300.

16. Müller, P. \& Scarsini, M. (2001). Stochastic comparisons of random vectors with a common copula. Mathematics of Operations Research 26: 723-740.

17. Nelsen, R.B. (1998). An introduction to copulas. New York: Springer-Verlag.

18. Rojo, J. \& He, G.Z. (1991). New properties and characterizations of the dispersive ordering. Statistics and Probability Letters 11: 365-372.

19. Saunders, I.W. \& Moran, P.A.P. (1978). On quantiles of the gamma and $F$ distributions. Journal of Applied Probability 15: 426-432.

20. Shaked, M. \& Shanthikumar, J.G. (1994). Stochastic orders and their applications. San Diego, CA: Academic Press.

21. Shaked, M. \& Shanthikumar, J.G. (1998). Two variability orders. Probability in the Engineering and Informational Sciences 12: 1-23.

22. Shaked, M. \& Wong, T. (1995). Preservation of stochastic orderings under random mapping by point processes. Probability in the Engineering and Informational Sciences 9: 563-580.

23. Sklar, A. (1959). Functions de répartition à $n$ dimensions et leurs marges. Publication du Institute Statistics du Universite de Paris 8: 229-231. 